\title{
Profile of ipilimumab and its role in the treatment of metastatic melanoma
}

\author{
This article was published in the following Dove Press journal: \\ Drug Design, Development and Therapy \\ 17 December 201I \\ Number of times this article has been viewed
}

\author{
Sapna P Patel \\ Scott E Woodman \\ Melanoma Medical Oncology \\ Department, University of Texas, \\ MD Anderson Cancer Center, \\ Houston, TX, USA
}

Correspondence: Scott E Woodman Melanoma Medical Oncology Department, University of Texas, MD Anderson Cancer Center, South Campus Research Building, SCR 2.3022, 7455 Fannin St, Houston, TX 77054, USA

Tel + I 7I35639286

Fax + I 7135633424

Emailswoodman@mdanderson.org

\begin{abstract}
Melanoma is an immunogenic cancer. However, the ability of the immune system to eradicate melanoma tumors is affected by intrinsic negative regulatory mechanisms. Multiple immune-modulatory therapies are currently being developed to optimize the immune response to melanoma tumors. Two recent Phase III studies using the monoclonal antibody ipilimumab, which targets the cytotoxic T-lymphocyte antigen (CTLA-4), a negative regulator of T-cell activation, have demonstrated improvement in overall survival of metastatic melanoma patients. This review highlights the clinical trial data that supports the efficacy of ipilimumab, the immune-related response criteria used to evaluate clinical response, and side-effect profile associated with ipilimumab treatment.
\end{abstract}

Keywords: ipilimumab, melanoma, T-cells, CTLA-4

\section{Introduction}

It has long been understood that among cancer types, melanoma is potentially treatable using immunologically-based therapies. Infiltrating lymphocytes are often present in tumors, demonstrating an immune response to melanoma tumors. However, infiltrating T-cells fail to be sufficiently activated to result in tumor reduction. Methods to enhance the T-cell response to melanoma tumors have shown promise. Treatment of metastatic melanoma with high dose interleukin-2 (IL-2), which stimulates T-cell activity, results in objective response rates of approximately $15 \%$ and durable complete response rates in up to $6 \%$ of cases. ${ }^{1,2}$ Adoptive T-cell Transfer (ACT), in which tumor-reactive lymphocytes are stimulated ex vivo and expanded before being re-introduced into the patient, has shown objective response rates of $50 \%-70 \%{ }^{3,4}$ Recent data indicates that ACT using lymphodepleting preparative regimens can result in durable complete responses in up to $\sim 20 \%$ of patients in a highly selected population. ${ }^{5}$ Finally, a fully human IgG4 antibody that blocks programmed death 1 (PD-1) inhibitory receptor on activated T-cells has shown encouraging results in early clinical trials, with 15 of 46 evaluable melanoma patients achieving objective responses in a Phase II study. ${ }^{6}$ All of these patients remained on trial at the time of presentation, suggesting potential durable responses are obtainable with anti-PD-1 treatment. Despite the potential of enhancing the native immune response to metastatic melanoma tumors, it is clear that there are complexities of the immune response that thwart the ability of T-cells to sufficiently attack melanoma tumors in most patients.

T-cell activation requires costimulatory signals. Melanoma antigens that are bound to the major histocompatibility complex (MHC) on antigen-presenting cells 
(APCs) require the costimulation of $\mathrm{CD} 28$ receptor on T-cells by CD80 or CD86 ligands on APCs for T-cell activation (Figure 1). The cytotoxic T-lymphocyte antigen-4 (CTLA-4) can bind with greater affinity to CD80 and CD86, and thus disrupt the necessary costimulatory signal provided by APCs. This led to the hypothesis that blockade of CTLA-4 function may allow for optimal costimulation of CD28 receptors on T-cells by APC CD80/86, and enhanced T-cell activation. Ipilimumab (Yervoy ${ }^{\mathrm{TM}}$ Bristol-Myers Squibb, New York, $\mathrm{NY}$ ) is a recombinant human $\mathrm{IgG} 1 \kappa$ monoclonal antibody that binds to CTLA-4 and blocks binding to CD80 or CD86 on APCs. Multiple elegant pre-clinical and early phase clinical studies demonstrated the proof of principle of this approach, ${ }^{7,8}$ and ipilimumab is now the first treatment in a randomized study to demonstrate a clear overall survival benefit in metastatic melanoma. ${ }^{9}$

\section{Pharmacology Mechanism of action}

Ipilimumab belongs to a class of immunomodulatory agents which alters the inherent balance of the immune system. It is a monoclonal antibody targeting the immune protein cytotoxic
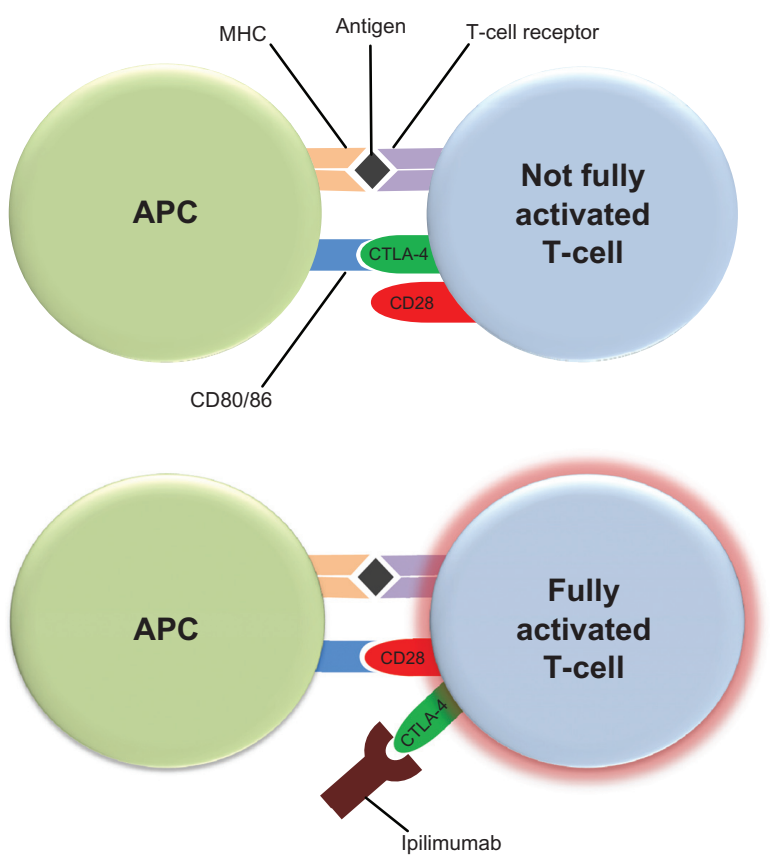

Figure I Ipilimumab blocks the costimulatory signal required for T-cell activation. Antigen-presenting cells (APCs) present melanoma antigens bound to the major histocompatibility complex (MHC) to T-cells. Costimulation of CD28 receptor on T-cells by CD80 or CD86 ligands on APCs is also required for optimal T-cell activation. The cytotoxic T-lymphocyte antigen-4 (CTLA-4) on T-cells can bind with greater affinity to CD80 and CD86, and thus disrupt the necessary costimulatory signal provided by APCs. Ipilimumab binds to CTLA-4 and blocks its binding to CD80 or CD86 on APCs allowing for costimulation of CD28 receptors on T-cells by APC CD80/86, and optimal T-cell activation. 9,10
T-lymphocyte antigen (CTLA-4). CTLA-4 is a negative regulator of T-cell activation and is expressed on activated T-cells as well as on T-regulatory cells. When T-cells bind to APCs, a costimulatory signal is needed to potentiate T-cell activation. This costimulatory signal takes the form of CD28, present on the T-cell, binding to the B7 family of receptors expressed by APC. CTLA-4 is also capable of binding to $\mathrm{B} 7$ receptors and, in doing so, inhibits costimulation and activation of T-cells. ${ }^{10,11}$ CTLA-4 knockout mice universally experience a fatal syndrome of lymphoproliferation which provides evidence for the key function of CTLA-4 as a negative regulator of the immune system. ${ }^{12-14}$ Interestingly, blockade of CTLA-4 does not lead to nonspecific T-cell activation but it does appear to augment immune responses in mice. ${ }^{11}$ Ipilimumab was developed with a transgenic murine model to create a monoclonal antibody with human immunoglobulin genes that binds CLTA-4, and blocks its interactions with B7. ${ }^{15,16}$

\section{Physical properties}

Ipilimumab is a fully human monoclonal antibody with two heavy chains and two kappa light chains linked together by way of disulfide bonds. The molecular weight is approximately $148 \mathrm{kDa}$ and it exists in solution at a physiologic $\mathrm{pH}$ of 7.0 .

\section{Pharmacokinetics}

Pharmacokinetic data was originally generated in mouse and cynomolgus monkey models. ${ }^{15,17}$ After infusion of a $10 \mathrm{mg} / \mathrm{kg}$ dose, the steady-state volume of distribution of ipilimumab was similar to the plasma volume of ipilimumab in cynomolgus monkeys. This implies that ipilimumab remains within the vasculature and does not undergo tissue distribution. ${ }^{18}$ Ipilimumab is systemically cleared and is not affected by hepatic or renal organ function in patients with a creatinine clearance of $\geq 29 \mathrm{~mL} /$ minute. ${ }^{19}$ Additionally, it is not metabolized via cytochrome $\mathrm{P} 450$ pathways; it is expected to undergo degradation into small peptides and amino acids and undergo excretion via normal protein catabolism. The half-life of the drug is 14.7 days and steady state is established by the third dose when administered every 3 weeks. Pursuant to these studies, a Phase I trial evaluated ipilimumab at $0.3 \mathrm{mg} / \mathrm{kg}, 1 \mathrm{mg} / \mathrm{kg}$, and $3 \mathrm{mg} / \mathrm{kg}{ }^{20,21}$

\section{Clinical applications Phase I trials}

The first human clinical studies were initiated at doses of $0.3,1$, and $3 \mathrm{mg} / \mathrm{kg}$ and studied across a variety of 
solid tumors. ${ }^{21-23}$ The $3 \mathrm{mg} / \mathrm{kg}$ dose was selected for further testing in humans based upon trough levels. Early studies did not establish a maximum tolerated dose and further clinical studies pushed the dose up to $10 \mathrm{mg} / \mathrm{kg}$. Nonetheless, doselimiting toxicities were noted in nearly all early studies of ipilimumab. ${ }^{21,24}$ These studies also noted that multiple-dose induction regimens given every 3 weeks improved disease control rates over single-dose induction regimens. ${ }^{25}$ The most notable responses were seen repeatedly in metastatic melanoma.

\section{Phase II trials in melanoma demonstrate dose-dependent responsiveness}

In a Phase II dose-finding study of ipilimumab in metastatic melanoma, responses were noted in a dose-dependent fashion when ipilimumab was dosed at $0.3 \mathrm{mg} / \mathrm{kg}, 3 \mathrm{mg} / \mathrm{kg}$, and $10 \mathrm{mg} / \mathrm{kg}$ every 3 weeks for four doses and analyzed using a statistical trend test. ${ }^{26}$ Ipilimumab was noted to be clinically active at doses of $3 \mathrm{mg} / \mathrm{kg}$ and higher. In a Phase II study of ipilimumab at $3 \mathrm{mg} / \mathrm{kg}$ every 4 weeks for four doses alone or in combination with dacarbazine $250 \mathrm{mg} / \mathrm{m}^{2} /$ day for 5 days for up to six courses, there was no statistically significant difference in response rate between the two arms (5.4\% vs $14.3 \%$, respectively). ${ }^{27}$ Without a significant increase in toxicity, further studies of ipilimumab at $10 \mathrm{mg} / \mathrm{kg}$ showed best overall response rates by WHO modified criteria in the range of $5.8 \%-15.8 \% .^{28,29}$ An ongoing Phase II single-institution trial (NCT01119508) seeks to further evaluate ipilimumab $10 \mathrm{mg} / \mathrm{kg}$ in combination with temozolomide. The primary endpoint of this study is the rate of 6-month progression-free survival with a secondary endpoint of overall response rate. These Phase II studies also evaluated the use of ipilimumab in a maintenance setting after four doses of induction ipilimumab. Patients who did not experience progressive disease after the induction period or toxicity requiring discontinuation of therapy were treated every 12 weeks in a maintenance setting and were continued on therapy until disease progression or unmanageable toxicity occurred ( $9 \%-20 \%$ of patients).

\section{Phase III trial shows improvement in overall survival in metastatic melanoma}

The largest Phase III study completed to date with ipilimumab enrolled 676 patients with previously treated metastatic melanoma and HLA-A*0201-positive class I major histocompatibility complex proteins. The reason for HLA status prerequisite was due to the presence of glycoprotein 100 (gp100) vaccine arms. Gp100 is one of the most common of the melanosomal proteins and is highly immunogenic. Its ability to stimulate tumor-reactive T-cells has been well-described and it was hypothesized that the addition of gp100 peptide vaccine to ipilimumab may enhance T-cell regulation and thus response rate compared with ipilimumab alone. ${ }^{30,31}$ The gp100 epitope is presented to the immune system in the context of HLA-A*0201 restricted peptides. As a result, patients were randomized in a 3:1:1 fashion to receive either ipilimumab $3 \mathrm{mg} / \mathrm{kg}$ plus gp100 peptide vaccine $(\mathrm{n}=403)$, ipilimumab $3 \mathrm{mg} / \mathrm{kg}$ alone $(\mathrm{n}=137)$, or gp100 vaccine alone $(\mathrm{n}=136)$. A retrospective analysis of previously published ipilimumab trials in metastatic melanoma noted similar median overall survival rates regardless of HLA status. ${ }^{32}$ Treatment was administered every 3 weeks for four doses in an induction setting. The primary endpoint of this study was initially designed to evaluate best overall response rate. After emergence of the Phase II data, and in coordination with another Phase III study, the primary endpoint was amended to overall survival (OS). For patients in the ipilimumab arms (alone or with gp100), median OS was 10.1 and 10.0 months, respectively, which was statistically significant compared to median OS of 6.4 months in the gp100 vaccine arm. This study allowed for re-induction ipilimumab in the originally assigned treatment regimen in patients who completed four doses of induction therapy and experienced stable disease or better at week 12 with subsequent disease progression. Thirty-one patients were treated with re-induction ipilimumab (23 with ipilimumab plus gp100 vaccine; eight with ipilimumab alone); 21 patients $(67.7 \%)$ achieved a response or stable disease with re-induction. On March 25, 2011, on the basis of this Phase III study, the US Food and Drug Administration (FDA) approved ipilimumab at a dose of $3 \mathrm{mg} / \mathrm{kg}$ for four induction doses to treat patients with metastatic melanoma. Due to previously published work, which noted no difference in overall survival or response rate based on HLA status, results of the Phase III trial can be extrapolated to ipilimumab-treated HLA-A*0201-negative patients. ${ }^{32}$ Another large, international, multi-center Phase III trial (NCT00324155) recently closed to enrollment and sought to compare OS, progression-free survival, and best overall response rate in dacarbazine plus ipilimumab $10 \mathrm{mg} / \mathrm{kg}$ vs dacarbazine plus placebo. Eligible patients $(n=502)$ were treated in the front-line metastatic setting with ipilimumab plus dacarbazine or placebo plus dacarbazine every 3 weeks for four doses followed by maintenance therapy. Although the overall response rate was not notably different between the two groups, the cohort receiving ipilimumab plus dacarbazine had a statistically significant improvement in the primary endpoint of OS compared to placebo plus dacarbazine (11.2 months vs 9.1 months). ${ }^{33}$ 


\section{Immune-related response criteria}

Patterns of response to immunotherapy such as ipilimumab have historically varied from traditional responses seen with classical cytotoxic therapies. Stabilization of disease or slow regressions of tumors are expected responses; in addition, responses to ipilimumab have been demonstrated after initial tumor flare or tumor inflammation resulting in progressive disease. Likewise, the development of new lesions, while traditionally considered criteria for termination of other treatments, does not always preclude a decrease in overall tumor burden in patients treated with ipilimumab. As such, traditional response criteria (WHO or RECIST) may underestimate the late responses seen with ipilimumab and deem treatment a clinical failure. The immunotherapy community, led by the Cancer Immunotherapy Consortium, previously the Cancer Vaccine Consortium, thereby led a process to review responses to immune-based treatments and establish new criteria which account for delayed response to therapy. This innovative set of criteria is known as immunerelated response criteria (irRC) and is a derivation of WHO criteria (Table 1). ${ }^{34}$ Approximately $8 \%-10 \%$ of patients evaluated in the early Phase II ipilimumab trials had their responses changed from progressive disease to immune-related partial response using irRC. ${ }^{28,29}$ The Phase III studies of ipilimumab in metastatic melanoma established irRC as the method of response evaluation.

\section{Safety profile}

\section{Immune-related adverse events}

Ipilimumab induces autoimmune-like adverse events in treated patients. Across early studies, the most commonly encountered immune-mediate effects were dermatitis, hepatitis, enterocolitis, hypophysitis, and uveitis. ${ }^{24,25}$ These effects are termed immune-related adverse events (irAEs). They appear most often during the induction phase of ipilimumab and were

Table I Immune-related response criteria

\begin{tabular}{ll}
\hline $\begin{array}{l}\text { Complete } \\
\text { response }\end{array}$ & $\begin{array}{l}\text { Disappearance of all lesions in two consecutive } \\
\text { observations at least } 4 \text { weeks apart } \\
\text { Partial }\end{array}$ \\
$\begin{array}{l}\geq 50 \% \text { decrease in tumor burden compared with } \\
\text { response }\end{array}$ & $\begin{array}{l}<50 \% \text { decrease in tumor burden compared with } \\
\text { btable }\end{array}$ \\
disease & $\begin{array}{l}\text { baseline and }<25 \% \text { increase in tumor burden } \\
\text { compared with nadir }\end{array}$ \\
Progressive & $\geq 25 \%$ increase in tumor burden compared with \\
disease & nadir in two consecutive observations at least \\
& 4 weeks apart \\
New & Do not automatically define progression; incorporated \\
lesions & into tumor burden
\end{tabular}

dose-related in early dose-escalation studies. ${ }^{26}$ Up to $80 \%$ of patients on clinical studies of ipilimumab reported irAEs, and $10 \%-17 \%$ of these are grade 3 or higher. The median time to resolution of immune-related adverse events was evaluated in one study and determined to be 6.3 weeks. In the Phase III trial of ipilimumab and gp100, there were 14 deaths overall; eight in the ipilimumab plus gp100 arm, four in the ipilimumab alone arm, and two in the gp100 alone arm. Seven of these 14 were considered to be immune-related and the deaths which have been attributed to ipilimumab involved the gastrointestinal tract (bowel perforation, enterocolitis, liver failure) and the nervous system (Guillain Barré syndrome). The FDA was tasked with balancing safety and adverse events of ipilimumab and created a Risk Evaluation and Mitigation Strategy (REMS) program for ipilimumab upon approval. This program educates prescribing healthcare professionals about the risk of potentially severe irAEs that can occur while using ipilimumab.

\section{Correlation between immune-related adverse events and response}

Several studies have explored the theory that occurrence of irAE correlates with disease response. The earliest findings noted that patients with autoimmune toxicity were more likely to have tumor regression than patients who did not exhibit autoimmune toxicity $(P=0.008) .{ }^{23}$ In the Phase I/II study by Weber et al, all four objective responders also had an irAE..$^{24}$ Additionally, 13 of 14 (93\%) patients in this study who experienced stable disease had an irAE. Although the rate of irAE of any grade was $76 \%$, there was no significant correlation between irAEs and response in this study. On further analysis, there was a significant correlation between grade 3 or 4 irAE and response in patients receiving ipilimumab at $10 \mathrm{mg} / \mathrm{kg}(P=0.03)$. Another study found that patients with objective responses were noted to have more severe irAEs, and this finding was significant $(P=0.0004){ }^{35}$ Although many patients with grade 0-2 irAEs experience clinical benefit either via disease stabilization or tumor regression, the disease control rate is higher in patients with grade 3-4 irAEs. ${ }^{29}$ It should be noted that the correlation between severe irAEs and response is most notable when ipilimumab is administered at $10 \mathrm{mg} / \mathrm{kg}$ and this correlation was not described in the Phase III trial which administered ipilimumab at $3 \mathrm{mg} / \mathrm{kg}$.

\section{Side effect management}

Bristol-Myers Squibb has developed guidelines to assist health care professionals in the management of immune-mediated 
adverse reactions based upon affected body region. ${ }^{36}$ Gastrointestinal toxicity is frequently cited as an irAE that requires management and manifests as diarrhea or enterocolitis. If symptoms are considered moderate, 4-6 stools per day over baseline or associated with abdominal pain, blood or mucus in the stool, ipilimumab should be withheld and antidiarrheal medications utilized until symptoms regress to at least a grade 1 . If severe or life-threatening symptoms are present, $\geq 7$ stools per day over baseline or associated with fever, ileus, or suspected bowel perforation, ipilimumab should be permanently discontinued and corticosteroids initiated at $1-2 \mathrm{mg} / \mathrm{kg}$ /day prednisone or methylprednisolone tapered slowly over 1 month. Concurrently, consideration of endoscopy is recommended, when appropriate. Systemic steroids should also be considered for the patient experiencing moderate gastrointestinal effects whose symptoms do not respond after 1 week of supportive care.

For patients with immune-mediated dermatitis, nonlocalized rash or diffuse rash $\leq 50 \%$ of skin surface can be managed by withholding ipilimumab and dispensation of topical or systemic corticosteroids. For severe dermatological effects such as Stevens-Johnson syndrome, toxic epidermal necrolysis, necrotic, bullous, or hemorrhagic desquamation, or full-thickness ulceration, ipilimumab should be permanently discontinued and systemic corticosteroids administered at $1-2 \mathrm{mg} / \mathrm{kg} /$ day of prednisone equivalent.

Management of other irAEs such as endocrinopathies driven by hypophysitis, neuropathies, respiratory and ocular manifestations, and hepatitis are also outlined in supplemental information provided by Bristol-Myers Squibb. In general, moderate irAEs can often be managed by withholding ipilimumab with or without supportive care while severe or lifethreatening irAEs often warrant permanent discontinuation of ipilimumab along with concurrent immunosuppressive therapy, most often in the form of systemic corticosteroids.

\section{Conclusion}

Melanoma is an immunogenic cancer, however, the immune system lacks the inherent capacity to eradicate melanoma tumors in most cases. Methods to enhance the ability of the immune system to attack melanoma have traditionally had marginal success, and served to remind us of the immense complexity of the balance between activating and inhibiting regulators of the immune system. Thus, it is noteworthy that the first randomized study to demonstrate a clear overall survival benefit in metastatic melanoma uses an immune-modulating agent. The effect of ipilimumab on metastatic melanoma is very encouraging and presents many intriguing further questions.
The use of ipilimumab has reinforced the need for oncologists to evaluate "tumor response" in a different manner than that used when using chemotherapeutic agents. Clinical responses can take months to appear given the need for an immune response to self-antigen to develop. Thus criteria, as presented in this review, are now being proposed to account for the difference seen when using immunologically-based therapies. The nature of response criteria will certainly have to be reviewed as new immune-based agents and combination therapies are developed.

Apart from the increase in median survival (approximately 4 months) seen with the use of ipilimumab, is the very encouraging durable complete responses ( $>5$ years) that have been observed in some of the earliest melanoma patients to be treated with ipilimumab. It is clear that immunotherapies (eg, high dose IL-2, adoptive T-cell transfer, anti-PD-1, and anti-CTLA-4) have the capacity to induce a durable complete response in a small subset of patients. It is unclear if patients who have a durable response to one immunotherapy would be more likely to have the same response to another immunotherapy, or if different immune-modulating agents can have a beneficial effect in different populations of patients. The development of markers that predict which patients will respond to treatment and which will not, will also serve to optimize the use of these immune-modulating agents while limiting unnecessary toxicity.

The identification of activating gene mutations (eg, $B R A F$, NRAS, KIT, GNAQ, GNA11) in different subtypes of melanoma has created the opportunity to identify and inhibit activated growth and survival signaling pathways within melanoma tumor cells..$^{37,38}$ It will be intriguing to see if the combination of immunotherapies with agents that target particular signaling pathways will enhance clinical response and overall survival, and most importantly long-term survival. Results from recent clinical trials have shown the efficacy of using small molecule inhibitors to target particular signaling pathway molecules. Vemurafenib, a BRAF mutant specific inhibitor recently gained FDA approval for use in advanced melanoma harboring the V600 mutation. ${ }^{39}$ Multiple reports have demonstrated clinical responses using a variety of tyrosine kinase inhibitors (imatinib, dasatinib, sunitinib) in KIT mutant melanoma. ${ }^{40-43}$ Likewise, early reports suggest that small molecule MEK inhibitors that are currently being evaluated in clinical trials may have efficacy in different subtypes of melanoma. ${ }^{44}$

The recent FDA-approval of ipilimumab for unresectable or metastatic melanoma is a major advance in the treatment of melanoma, and will undoubtedly serve as the foundation of future treatment regimens. 


\section{Disclosure}

The authors report no conflicts of interest in this work.

\section{References}

1. Atkins MB, Lotze MT, Dutcher JP, et al. High-dose recombinant interleukin 2 therapy for patients with metastatic melanoma: analysis of 270 patients treated between 1985 and 1993. J Clin Oncol. 1999;17(7):2105-2116.

2. Smith FO, Downey SG, Klapper JA, et al. Treatment of metastatic melanoma using interleukin-2 alone or in conjunction with vaccines. Clin Cancer Res. 2008;14(17):5610-5618.

3. Dudley ME, Yang JC, Sherry R, et al. Adoptive cell therapy for patients with metastatic melanoma: evaluation of intensive myeloablative chemoradiation preparative regimens. J Clin Oncol. 2008;26(32):5233-5239.

4. Besser MJ, Shapira-Frommer R, Treves AJ, et al. Clinical responses in a phase II study using adoptive transfer of short-term cultured tumor infiltration lymphocytes in metastatic melanoma patients. Clin Cancer Res. 2010;16(9):2646-2655.

5. Rosenberg SA, Yang JC, Sherry RM, et al. Durable complete responses in heavily pretreated patients with metastatic melanoma Using $\mathrm{T}$ cell transfer immunotherapy. Clin Cancer Res. 2011;17(13):4550-4557.

6. Sznol M, Powderly JD, Smith DC, et al. Safety and antitumor activity of biweekly MDX-1106 (Anti-PD-1, BMS-936558/ONO-4538) in patients with advanced refractory malignancies [abstract]. J Clin Oncol. 2010;28(15s):2506.

7. Weber J. Immune checkpoint proteins: a new therapeutic paradigm for cancer - preclinical background: CTLA-4 and PD-1 blockade. Semin Oncol. 2010;37(5):430-439.

8. Curran MA, Montalvo W, Yagita H, Allison JP. PD-1 and CTLA-4 combination blockade expands infiltrating $\mathrm{T}$ cells and reduces regulatory $\mathrm{T}$ and myeloid cells within B16 melanoma tumors. Proc Natl Acad Sci US A. 2010;107(9):4275-4280.

9. Hodi FS, O’Day SJ, McDermott DF, et al. Improved survival with ipilimumab in patients with metastatic melanoma. $N$ Engl J Med. 2010;363(8):711-723

10. Lee KM, Chuang E, Griffin M, et al. Molecular basis of T cell inactivation by CTLA-4. Science. 1998;282(5397):2263-2266.

11. Egen JG, Kuhns MS, Allison JP. CTLA-4: new insights into its biological function and use in tumor immunotherapy. Nat Immunol. 2002;3(7):611-618

12. Tivol EA, Borriello F, Schweitzer AN, Lynch WP, Bluestone JA, Sharpe AH. Loss of CTLA-4 leads to massive lymphoproliferation and fatal multiorgan tissue destruction, revealing a critical negative regulatory role of CTLA-4. Immunity. 1995;3(5):541-547.

13. Waterhouse P, Penninger JM, Timms E, et al. Lymphoproliferative disorders with early lethality in mice deficient in Ctla-4. Science. 1995;270(5238):985-988.

14. Chambers CA, Sullivan TJ, Allison JP. Lymphoproliferation in CTLA4-deficient mice is mediated by costimulation-dependent activation of CD4+ T cells. Immunity. 1997;7(6):885-895.

15. Keler T, Halk E, Vitale L, et al. Activity and safety of CTLA-4 blockade combined with vaccines in cynomolgus macaques. J Immunol. 2003;171(11):6251-6259.

16. Morse MA. Technology evaluation: ipilimumab, Medarex/BristolMyers Squibb. Curr Opin Mol Ther. 2005;7(6):588-597.

17. Palmisano GL, Tazzari PL, Cozzi E, et al. Expression of CTLA-4 in nonhuman primate lymphocytes and its use as a potential target for specific immunotoxin-mediated apoptosis: results of in vitro studies. Clin Exp Immunol. 2004;135(2):259-266.

18. Anonymous (Study No. DS06064: BMS-663513 and BMS-734016 (MDX-010): One-month Intravenous Combination Toxicity Study in Monkeys. Bristol-Myers Squibb Company, 2007.

19. Kohn DB, Dotti G, Brentjens R, et al. CARs on track in the clinic. Mol Ther. 2011;19(3):432-438.
20. Sanderson K, Scotland R, Lee P, et al. Autoimmunity in a phase I trial of a fully human anti-cytotoxic T-lymphocyte antigen-4 monoclonal antibody with multiple melanoma peptides and Montanide ISA 51 for patients with resected stages III and IV melanoma. J Clin Oncol. 2005;23(4):741-750.

21. Maker AV, Phan GQ, Attia P, et al. Tumor regression and autoimmunity in patients treated with cytotoxic T lymphocyte-associated antigen 4 blockade and interleukin 2: a phase I/II study. Ann Surg Oncol. 2005;12(12):1005-1016.

22. Phan GQ, Yang JC, Sherry RM, et al. Cancer regression and autoimmunity induced by cytotoxic T lymphocyte-associated antigen 4 blockade in patients with metastatic melanoma. Proc Natl Acad Sci US A. 2003;100(14):8372-8377.

23. Attia P, Phan GQ, Maker AV, et al. Autoimmunity correlates with tumor regression in patients with metastatic melanoma treated with anti-cytotoxic T-lymphocyte antigen-4. J Clin Oncol. 2005;23(25):6043-6053.

24. Weber JS, O'Day S, Urba W, et al. Phase I/II study of ipilimumab for patients with metastatic melanoma. J Clin Oncol. 2008;26(36): 5950-5956.

25. Maker AV, Yang JC, Sherry RM, et al. Intrapatient dose escalation of anti-CTLA-4 antibody in patients with metastatic melanoma. J Immunother. 2006;29(4):455-463.

26. Wolchok JD, Neyns B, Linette G, et al. Ipilimumab monotherapy in patients with pretreated advanced melanoma: a randomised, double-blind, multicentre, phase 2, dose-ranging study. Lancet Oncol. 2010;11(2):155-164.

27. Hersh EM, O'Day SJ, Powderly J, et al. A phase II multicenter study of ipilimumab with or without dacarbazine in chemotherapy-naive patients with advanced melanoma. Invest New Drugs. 2011;29(3):489-498.

28. O'Day SJ, Maio M. Chiaron-Sileni V, et al. Efficacy and safety of ipilimumab monotherapy in patients with pretreated advanced melanoma: a multicenter single-arm phase II study. Ann Oncol. 2010;21(8): 1712-1717.

29. Weber J, Thompson JA, Hamid O, et al. A randomized, double-blind, placebo-controlled, phase II study comparing the tolerability and efficacy of ipilimumab administered with or without prophylactic budesonide in patients with unresectable stage III or IV melanoma. Clin Cancer Res. 2009;15(17):5591-5598.

30. Parkhurst MR, Salgaller ML, Southwood S, et al. Improved induction of melanoma-reactive CTL with peptides from the melanoma antigen gp100 modified at HLA-A*0201-binding residues. J Immunol. 1996;157(6):2539-2548.

31. Kawakami Y, Robbins PF, Wang X, et al. Identification of new melanoma epitopes on melanosomal proteins recognized by tumor infiltrating $\mathrm{T}$ lymphocytes restricted by HLA-A1, -A2, and -A3 alleles. J Immunol. 1998;161(12):6985-6992.

32. Wolchok JD, Weber JS, Hamid O, et al. Ipilimumab efficacy and safety in patients with advanced melanoma: a retrospective analysis of HLA subtype from four trials. Cancer Immun. 2010;10:9.

33. Robert C, Thomas L, Bondarenko I, et al. Ipilimumab plus dacarbazine for previously untreated metastatic melanoma. $N$ Engl J Med. 2011;364(26):2517-2526

34. Wolchok JD, Hoos A, O'Day S, et al. Guidelines for the evaluation of immune therapy activity in solid tumors: immune-related response criteria. Clin Cancer Res. 2009;15(23):7412-7420.

35. Downey SG, Klapper JA, Smith FO, et al. Prognostic factors related to clinical response in patients with metastatic melanoma treated by CTL-associated antigen-4 blockade. Clin Cancer Res. 2007;13(22 Pt 1): 6681-6688.

36. Anonymous YERVOY ${ }^{\mathrm{TM}}$ (ipilimumab) Immune-mediated Adverse Reaction Management Guide. FDA-approved REMS. Available at: http:// www.yervoy.com/hcp/index.aspx. Accessed November 23, 2011.

37. Flaherty KT, Fisher DE. New strategies in metastatic melanoma: oncogene-defined taxonomy leads to therapeutic advances. Clin Cancer Res. 2011;17(15):4922-4928.

38. Woodman SE, Davies MA. Targeting KIT in melanoma: a paradigm of molecular medicine and targeted therapeutics. Biochem Pharmacol. 2010;80(5):568-574. 
39. Flaherty KT, Puzanov I, Kim KB, et al. Inhibition of Mutated, Activated BRAF in Metastatic Melanoma. N Engl J Med. 2010;363(9):809-819.

40. Carvajal RD, Antonescu CR, Wolchok JD, et al. KIT as a therapeutic target in metastatic melanoma. JAMA. 2011;305(22):2327-2334.

41. Woodman SE, Trent JC, Stemke-Hale K, et al. Activity of dasatinib against L576P KIT mutant melanoma: molecular, cellular, and clinical correlates. Mol Cancer Ther. 2009;8(8):2079-2085.

42. Kluger HM, Dudek AZ, McCann C, et al. A phase 2 trial of dasatinib in advanced melanoma. Cancer. 2010;117(10):2202-2208.
43. Satzger I, Kuttler U, Volker B, Schenck F, Kapp A, Gutzmer R. Anal mucosal melanoma with KIT-activating mutation and response to imatinib therapy - case report and review of the literature. Dermatology. 2010;220(1):77-81.

44. Adjei AA, Cohen RB, Franklin W, et al. Phase I pharmacokinetic and pharmacodynamic study of the oral, small-molecule mitogen-activated protein kinase kinase 1/2 inhibitor AZD6244 (ARRY-142886) in Patients With Advanced Cancers. J Clin Oncol. 2008;26(13):2139-2146.

\section{Publish your work in this journal}

Drug Design, Development and Therapy is an international, peerreviewed open-access journal that spans the spectrum of drug design and development through to clinical applications. Clinical outcomes, patient safety, and programs for the development and effective, safe, and sustained use of medicines are a feature of the journal, which has also been accepted for indexing on PubMed Central. The manuscript management system is completely online and includes a very quick and fair peer-review system, which is all easy to use. Visit http://www.dovepress.com/testimonials.php to read real quotes from published authors.

\footnotetext{
Submit your manuscript here: http://www.dovepress.com/drug-design-development-and-therapy-journal
} 